Artículo 7/2012, noviembre (n. $\left.{ }^{\circ} 139\right)$

\title{
La necesidad de un informe social para la decisión y ejecución de las penas comunitarias
}

\section{Elena Larrauri Pijoan (Universidad Pompeu Fabra)}

Resumen: El texto sugiere la necesidad de contar con un 'informe

social' que aporte datos sobre la persona, su entorno social y su actitud frente al delito, antes de que el juez imponga una pena. Con apoyo en entrevistas a jueces y técnicos del sistema de justicia penal, el estudio plantea que la práctica hoy extendida en España de consultar sólo la antigüedad del hecho y la existencia de antecedentes penales conlleva que se impongan penas alternativas a la prisión que pueden ser de difícil cumplimiento. El informe social expone además variables dinámicas y permite recomendar el tipo de pena, los plazos y los deberes específicos que el condenado debe cumplir. Esta información, y un mejor conocimiento de la labor de los delegados de ejecución de penas y medidas alternativas, pueden ayudar a que los jueces asuman que las penas alternativas a la prisión no son sólo para las personas que delinquen por primera vez.

Palabras clave: informe social, penas alternativas, suspensión de la pena de prisión

Title: The need of a Presentence Report in the sentencing process of community sanctions

Abstract: This article tries to find out the reasons that explain the scarce use of Presentence Reports in the Spanish adult criminal justice system. It is based mainly on interviews to Spanish judges to understand why they hardly request such a report. After discussing these reasons the author argues that a Presentence Report is necessary in order to alert the judge about the existence of an appropriate community sentence, and in order to tailor the specific requirements of the community sentence to that offender.

Key words: Presentence Reports, Community Sanctions, Probation

Recepción del original: 17 julio 2012 Fecha de aceptación: 5 noviembre 2012

\section{Sumario: 1. Introducción. 2. Metodología. 3. Resultados. 4. Conclusiones y} recomendaciones

\section{Introducción}

En diversos países europeos (Reino Unido, Bélgica, Holanda, Dinamarca), además de Estados Unidos y Canadá, existe la posibilidad de solicitar un 'informe social' en el proceso penal, más conocido como Presentence Report (PSR), cuya función es la de proporcionar información al juez sobre: a) la situación personal y social de la persona acusada, b) su actitud frente al delito y c) el tipo de pena o medida que pueda ser más resocializadora.

Este informe se considera crucial para que el juez pueda imponer un castigo proporcional y adecuado, y así evitar una pena cuyo cumplimiento resulte imposible o inconveniente en las condiciones concretas de la persona. Por ejemplo: una pena de trabajos en beneficio de la comunidad (TBC) a quien por motivos de drogodependencia no puede cumplirla; la obligación de asistir a un programa de violencia de género a quien apenas tiene conocimientos de la lengua en que se imparte; una multa a quien está pasando una pensión a su ex cónyuge o a un extranjero en situación irregular en España que no dispone de permiso de residencia y trabajo en vigor para poder pagarla con ingresos legales; o una pena de expulsión a un extranjero proveniente de un país que sufre un conflicto bélico. El informe social permite dictar una pena adecuada a la situación social y características individuales de la persona y con ello facilitar el cumplimiento de las penas no privativas de libertad.

La segunda función del informe social es alertar y orientar a los jueces acerca de todas las posibilidades existentes de cumplir una pena en medio abierto. Por ello, este informe también se considera útil en Europa para aumentar el número de condenas a penas comunitarias y con ello reducir, quizás, el número de condenas a prisión. En este sentido se fija como objetivo del informe social sugerir una pena adecuada a la persona que evite su ingreso en prisión: una pena que pueda cumplirse y suministre el marco en el cual pueda producirse la rehabilitación.

Una tercera utilidad del informe social reside en recoger información sobre la persona que siente las bases para su posterior supervisión durante la ejecución de la pena y que permita afrontar sus necesidades y contrarrestar el riesgo de reincidir. Los informes sociales son pues especialmente necesarios en aquellas penas no privativas de libertad que imponen determinadas obligaciones a la persona condenada (como, por ejemplo, su participación en un trabajo a la comunidad, su asistencia a un programa formativo y su tratamiento en un programa de desintoxicación), pues permiten una mejor concreción de las obligaciones particulares y los plazos que la persona debe cumplir. En estas penas comunitarias, que no privan de libertad y someten al sujeto condenado a una supervisión por parte de técnicos del sistema de justicia penal, el informe social se considera el primer paso en base al cual se configura la posterior intervención penal en forma de entrevistas, seguimiento y asesoramiento por parte de los técnicos del sistema de justicia penal.

El informe social tiene como destinatarios los jueces penales. En algunos países este informe lo elabora el personal del sistema de justicia penal, en otros 
lo redactan trabajadores sociales de la administración local (Tata, 2010, 24), y en otros (por ejemplo, en Dinamarca) lo redactan estudiantes universitarios (Wandall, 2010, 334). En ocasiones este informe se escribe una vez que la persona ya ha aceptado su responsabilidad y se limita a presentar las diversas opciones de pena al juez; en otras se redacta antes del juicio y por tanto antes de que la culpabilidad esté demostrada, por lo que puede incidir no sólo en el tipo de pena más adecuada sino también en el juicio de culpabilidad, en atención a las circunstancias individuales de la persona.

En España este informe es obligatorio en el ámbito de la justicia juvenil (artículo 27 de la L.O. 5/2000) y todos los miembros del equipo técnico entrevistados en el marco de esta investigación insisten en su importancia para asesorar al juez en la pena que debe imponer, para orientar en la supervisión que se realiza del menor y para suministrar información dinámica sobre la ejecución de las penas. Evidentemente hay algunas diferencias entre el sistema penal juvenil y el de adultos, pero además de que estas no son, a mi juicio, concluyentes para descartar un informe social, debe recordarse que el sistema penal de adultos trata también, y de forma numerosa, con 'adultos' de 18 años.

En el sistema de justicia penal de adultos la necesidad de este informe es, o era, una idea compartida por el legislador español. El RD 515/2005, ahora derogado por el nuevo RD 840/2011, de 17 de junio, por el que se establecen las circunstancias de ejecución de las penas de trabajo en beneficio de la comunidad y de localización permanente en centro penitenciario, de determinadas medidas de seguridad, así como de la suspensión de la ejecución de la penas privativas de libertad y de sustitución de penas, había regulado su petición opcional (artículo 28). Sin embargo, que esta regulación se encontrara en un real decreto, que la petición del informe fuera discrecional en todos los casos, y quizás también el hecho de que no se explicitara exactamente quién y especialmente para qué debiera pedirse, ocasionó un gran desconocimiento -y una infrautilización- de este informe. Así por ejemplo, la tabla 1 muestra las cifras de los informes sociales redactados en Cataluña entre 2001 y 2011 por el Equip d'Assessorament Tècnic Penal (EATP), que es el equipo encargado de redactarlos a petición del juez de instrucción y del juez de ejecución.

\section{Metodología}

Si se tiene en cuenta que en Cataluña hubo en el año 201018.698 personas sometidas a supervisión en penas comunitarias, se puede apreciar la limitada incidencia del informe. Pero entonces, ¿̇a qué se debe la escasa solicitud de este recurso por parte de los jueces y fiscales españoles? Para averiguarlo se realizaron 15 entrevistas semi-estructuradas durante el mes de julio de 2009. El número total de jueces entrevistados representa aproximadamente el 50\% de cada una de las especialidades. Así, se realizaron dos entrevistas a jueces de violencia sobre la mujer (de un total de cuatro), nueve a jueces de lo penal (de un total de 23) y cuatro a jueces de ejecución penal (de un total de 8). Las entrevistas se llevaron a cabo por Lorena Antón y fueron transcritas por Patricia Martín. Además Elena Larrauri entrevistó a 5 miembros del Equip d'Assessorament Tècnic Penal (EATP) y a 4 del Equip Tècnic de Menors (ETM), las personas encargadas precisamente de asesorar a los jueces. A continuación se presentan los resultados de las entrevistas.

\section{Resultados}

Por lo que respecta a los concretos motivos aducidos por los jueces de instrucción para no pedir un informe social se alude en primer lugar a que la estructura de los juicios rápidos lo impide, alegando en segundo lugar la existencia en numerosos casos de una sentencia por conformidad. No obstante, pareciera que aun en juicios rápidos se podría pedir un breve informe que a veces se elabora exclusivamente en base a la entrevista con un técnico y que sin duda aportaría alguna información más respecto de la persona que se está juzgando. En cuanto a la conformidad, es razonable pensar que precisamente en estos casos en que el propio juez de instrucción decide acerca de la suspensión y especialmente de las reglas de conducta a imponer, también debería conocer las circunstancias individuales de la persona.

Las razones alegadas por el juez de lo penal consisten especialmente en que este informe debía ya constar en el expediente judicial que realiza el juez de instrucción, y que toda la información debe estar recogida 'antes del juicio oral', por lo que, en consecuencia, no le compete solicitar ningún informe social adicional. No obstante, además de un cierto desconocimiento acerca de la finalidad del informe, pareciera que el artículo 729 de la Ley de Enjuiciamiento Criminal permite al juez de lo penal solicitarlo de oficio cuando, por ejemplo, los indicios de alcoholismo surgen por primera vez en el transcurso del juicio oral. Un segundo motivo alegado por parte de los jueces de lo penal es que son los abogados quienes deben aportar esa información; sin embargo ello no es exacto, puesto que los abogados pueden aportarla, pero esta información de parte no debería sustituir la que el juez requiere para poder imponer la pena más adecuada.

Por último, en cuanto a los motivos específicos expuestos por los jueces de ejecución para no pedir los informes sociales la mayoría considera que estos deberían haber sido solicitados con anterioridad y, en consecuencia, son muy reticentes a tomar en cuenta lo que los delegados de ejecución advierten (por ejemplo, la necesidad de aplicar una suspensión especial por drogodependencias) si la sentencia no ha recogido este hecho (y la sentencia no lo

Tabla 1. Número de informes sociales en Cataluña 2001-2011

\begin{tabular}{|l|r|r|r|r|r|r|r|r|r|r|}
\hline Años & 2001 & 2002 & 2003 & 2004 & 2005 & 2006 & 2007 & 2008 & 2009 & 2010 \\
\hline Informes & 584 & 591 & 645 & 739 & 812 & 769 & 873 & 830 & 676 & 617 \\
\hline
\end{tabular}


plasma porque ni el juez de lo penal ni el de instrucción han solicitado informe social ni han detectado esta circunstancia por otros medios). La oposición de muchos jueces de ejecución a solicitar el informe social puede ser objetada argumentándose que los jueces de ejecución disponen de un ámbito de autonomía que la ley les concede precisamente para tomar decisiones, como por ejemplo suspender la pena de prisión y condicionarla al cumplimiento de diversas obligaciones (artículo 83 del código penal). Además, respecto de la problemática específica acerca de si puede concederse la suspensión extraordinaria por drogodependencia, debe advertirse que también cabe la interpretación de que la drogadicción ha de atenderse siempre y cuando quede acreditada en cualquier momento del proceso. $Y$ en tercer lugar puede apuntarse a que también el artículo 88 del código penal estipula diversos criterios, más allá de la existencia de antecedentes penales, que los jueces deben considerar para decidir acerca de la sustitución.

En general, de todas las entrevistas realizadas a los jueces, se desprende que hay algunos factores comunes que impiden la solicitud de un informe social:

1) Hay una confusión entre un informe médico forense destinado a analizar la culpabilidad de la persona y un informe social que pretende orientar sobre qué tipo de pena es adecuada. En tanto el primero es solicitado generalmente a los médicos forenses y tiene por finalidad ayudar a determinar la culpabilidad, el segundo es elaborado con carácter general por técnicos del sistema de justicia penal y suministra información sobre la situación social y la condición penal actual de la persona condenada. De esta manera el informe social pretende orientar de forma pragmática al juez sobre qué tipo de pena puede ser cumplida por la persona realmente (y eventualmente ayudarle a confrontar alguno de sus problemas que puedan estar relacionados con el delito). En tanto los jueces españoles conocen de la existencia del primer informe, el cual entienden que elaboran los médicos forenses antes del juicio oral, desconocen la posibilidad de solicitar el segundo, el cual se redacta generalmente por técnicos sociales después de confirmada la culpabilidad y en la fase de ejecución de la condena, como por ejemplo realiza el equipo de asesoramiento técnico (EATP) en Cataluña.

2) Hay también un cierto desconocimiento sobre qué juez debería pedir un informe social, producto quizás de la falta de reflexión sobre su finalidad. En la actualidad apenas se demandan informes sociales para decidir qué tipo de pena imponer. En general los únicos informes conocidos son los que pretenden incidir sobre el juicio de culpabilidad (cuánta pena). En estos casos, como ya hemos advertido, si el juez de instrucción no pide un informe ello produce efectos en cadena puesto que todos los jueces entienden que lo debería haber pedido 'otro'. Estos efectos perduran en el juicio porque el juez de lo penal desconoce una particular circunstancia individual de la persona que está siendo juzgada (por ejemplo, la drogodependencia), y en la ejecución de la sentencia porque la pena que se impone será luego más difícil de ejecutar.

3) Hay una realidad generalizada de excesiva carga de trabajo y un temor muy razonable de que la solicitud de unos informes sociales contribuya a sobrecargar el sistema. Ello junto con una actitud judicial 'neo-clásica' (Beyens y Scheirs, 2010), de cumplimiento estricto de la ley sin atender a las consecuencias de la pena y desconocimiento de la situación de la persona a la que se está juzgando, ayuda a entender por qué los jueces españoles no consideran necesario solicitar un informe social para un mejor conocimiento del entorno social de la persona acusada.

4) Existe por último una convicción en la jurisdicción penal de adultos de que el juez apenas goza de discrecionalidad. Este puede ser el caso en algunos supuestos en los que la ley impone una determinada medida, pero dista de ser cierto en la mayoría de las condenas en las que el juez dispone de un margen de actuación. A juicio de los jueces entrevistados 'la ley es muy clara' e indica qué pena debe dictarse. Sin embargo pareciera que los jueces disponen de una mayor discrecionalidad de la que ejercen. Ahora bien, cen base a qué información ejerce el juez su discrecionalidad?

5) En nuestras entrevistas se observa que los jueces examinan pocos datos para ejercer sus facultades en la elección del tipo de pena: los antecedentes penales, la gravedad y la antigüedad del hecho. En general los jueces consideran que ya disponen de la información más relevante o juzgan innecesario, más allá de la circunstancia relativa a algún tipo de drogodependencia, indagar sobre las características individuales de la persona, sus circunstancias sociales, su actitud frente al delito, su historial penal en penas anteriormente ejecutadas y el impacto del delito en la víctima. Ello no implica necesariamente que desconsideren toda información individual pero parece evidente que los jueces no se sienten responsables de tomar en consideración una información que facilite que la pena impuesta pueda finalmente ejecutarse.

\section{Conclusiones y recomendaciones}

Si bien está ampliamente admitida la necesidad de contar con informes para precisar el juicio de culpabilidad, los jueces entrevistados son escépticos acerca de la utilidad de solicitar informes para orientar acerca del tipo de pena. En mi opinión, abogar por introducir un informe social pretende, en última instancia, suministrar más información de variables dinámicas antes de imponer la condena, como, por ejemplo, datos socio-demográficos (si el sujeto está pagando una pensión o si está trabajando) y datos penales (si está ejecutándose otra pena), circunstancias que rodean el hecho (si se arrepiente, si ha intentado reparar el daño causado o si ha cambiado su situación desde que realizó el delito), así como las posibles manifestaciones de la víctima. La información suministrada por un equipo técnico sobre la persona acusada debería abarcar bastante más que los problemas de drogas y alcohol que ésta pueda tener, ya que 
permitiría especialmente capturar los denominados riesgos dinámicos.

La necesidad de contar con un informe social se hace más evidente a mi juicio en un sistema que, especialmente en las sucesivas reformas penales de 2003, 2004, 2007 y 2011, ha ido realizando una apuesta por penas que conllevan supervisión, que imponen reglas de conducta y que implican una actividad por parte de la persona condenada, como los trabajos en beneficio de la comunidad, o la participación en programas formativos.

La ausencia de información puede traer como consecuencia una falta de adecuación de las obligaciones impuestas y originar un incumplimiento y el procesamiento por un nuevo delito de quebrantamiento. Adicionalmente impide usar de forma más creativa todas las posibilidades existentes en el código penal para adecuar las posibles obligaciones y plazos a distintas exigencias retributivas y necesidades resocializadoras. En síntesis la ausencia de un informe social produce en numerosas ocasiones respuestas sólo 'formalmente' correctas y adecuadas.

En el supuesto de que el legislador español decidiera impulsar la solicitud de un informe social para decidir el tipo de pena, ¿qué juez, convencido de su utilidad, debería solicitarlo? El informe podría ser solicitado por el juez de instrucción. Ello permitiría que el juez de lo penal tuviera una mayor información sobre la persona en el momento de imposición de la condena. Sin embargo, si el informe es pedido por el juez de instrucción ello implica que la persona no ha sido todavía condenada (ni necesariamente ha aceptado su culpabilidad) y, por ello, como demuestra la experiencia del sistema penal juvenil, los técnicos son reacios en este caso a sugerir medidas concretas. En estos supuestos y, tal como hace el EATP de Barcelona en la actualidad, el informe se centra fundamentalmente en el cociente intelectual, el consumo de drogas y aspectos relativos a las circunstancias de la comisión del delito, lo cual permite que los jueces conozcan esta información antes de imponer la condena. A cambio puede verse como una limitación que el informe no realice recomendación alguna de pena.

En España es en la fase de ejecución cuando se decide qué tipo de pena ha de imponerse concretamente. En consecuencia es lógico también prever que el juez de ejecución (en las ciudades en que éste existe y, donde no, el juez de lo penal en el trámite de ejecución) pida estos informes antes de decidir si es posible suspender la pena de prisión impuesta; si impone algunas obligaciones y por cuánto tiempo; si concede la suspensión extraordinaria por drogodependencia; y si puede sustituir la pena de prisión por multa (pues la persona tiene dinero), por trabajos en beneficio de la comunidad o por localización permanente, pudiendo añadir además determinadas obligaciones del artículo 83. En estos casos, debido a que la responsabilidad ya ha sido declarada, el informe sugiere, como realiza el EATP de Barcelona, algún tipo de pena que pueda incidir sobre la problemática específica de la persona. Sin embargo, no solicitar informe alguno hasta este estadio conlleva el inconveniente detectado de la poca autonomía que algunos jueces de ejecución se atribuyen para tomar determinadas decisiones si no están reflejadas en la sentencia.

Es necesario recordar que el informe social cumple una función dual (Persson y Svensson, 2012): orienta en el tipo de pena y es el primer paso de inicio efectivo de la supervisión por parte de los delegados de ejecución de medidas (o servicios de gestión de penas y medidas alternativas). En la actualidad, aun cuando no se pida un informe social, el delegado de ejecución de penas y medidas alternativas, o el supervisor de la entidad donde se ejecuta la pena, sí poseen cierta información individual, pero ésta se obtiene de la entrevista que se realiza con la persona condenada una vez ya se ha concretado la pena por el juez de ejecución. Es entonces cuando se evalúa su grado de riesgo, se descubre su situación de drogodependencia o cualquier otra circunstancia individual referida por ejemplo a su situación familiar o integración socio-laboral. El problema es que en estos momentos la sentencia ya está ejecutándose y por tanto es difícil integrar lo desvelado en las entrevistas con los términos de la sentencia.

A modo de conclusión, los informes sociales pueden ser otro intento para conseguir el objetivo de que la pena de prisión sea un último recurso, de imposición sólo cuando todas las demás medidas han fracasado, al orientar y apoyar al juez en su búsqueda de una pena alternativa. Esta es la función que efectivamente cumplen en el sistema penal juvenil catalán, en el que el compromiso del equipo técnico de menores con el principio de internamiento como ultima ratio consigue en numerosas ocasiones que el ministerio fiscal cambie su petición de internamiento por la de medio abierto. No hay ninguna razón por la cual este modelo no pueda ser exportable al sistema penal de 'adultos' en el que un $42,60 \%$ de las personas condenadas tienen entre 18 y 30 años.

$\mathrm{El}$ informe social permite orientar al juez y plantearle alternativas viables sobre penas que pueden y deben ser cumplidas en medio abierto con una adecuada supervisión. En segundo lugar, puede permitir que el juez ajuste mejor la duración de la suspensión y las reglas de conducta asociadas a ésta, evitando que todas las decisiones judiciales sean formalmente idénticas y poco adecuadas al penado individual. Una mejor individualización de las condenas facultaría una supervisión específica a distintos tipos de infractores, lo cual redundaría probablemente en una mayor tasa de cumplimiento y una mejor reinserción. Para que esta individualización pueda ser realizada por el juez, en vez de por los servicios administrativos encargados de la ejecución de la pena, se requiere que aquél disponga de una información que le permita discernir qué infractores requieren de una supervisión más intensa y para cuáles es suficiente una mera suspensión. Por último, es de esperar que ello favorezca una mayor utilización de las medidas penales alternativas para ofensores de riesgo medio sin necesidad de recurrir a la pena de prisión. 


\section{Referencias (versión digital)}

\section{Bibliografía:}

ANTÓN, L. y LARRAURI, E. (2009) Violencia de género ocasional: un análisis de las penas ejecutadas, Revista Española de Investigación Criminológica, http://www.criminologia.net/reic.html

BLAY, E. (2007) Nueve tópicos acerca del trabajo en beneficio de la comunidad: la necesidad de una discusión basada en conocimientos empíricos. Indret 4/2007.

- (2011) El papel de los jueces en la ejecución de las penas comunitarias: una investigación empírica y algunas propuestas para la reflexión, en Larrauri, E. y Blay, E (coords) Las Penas comunitarias en Europa. Trotta, Madrid.

CACHÓN, M. y CID, J. (2003) Conformidad del acusado y penas alternativas a la prisión en los juicios rápidos. La Ley, número 5820, miércoles 9 de julio.

CID, J. (2009) La elección del castigo. Bosch, Barcelona.

- (2010) La política criminal europea en materia de sanciones alternativas a la prisión y la realidad española: una brecha que debe superarse, en Arangüena,C. (dir) Espacio Europeo de Libertad, seguridad y Justicia: últimos avances en cooperación judicial penal, Lex Nova, Valladolid.

DOWNING, K. y LYNCH, R. (1997) Pre-sentence reports: Does quality matter?, Social Policy and Administration, vol. 31, no. 2, pp. 173-190.

FIELD, S. y TATA, C. (2010) Connecting legal and social justice in the neo-liberal world? the construction, interpretation and use of pre-sentence reports, Punishment and Society, vol. 12, no. 3, pp. 235-238.

GIMENO SENDRA, V. (2007) Derecho procesal Penal, $2^{a}$ edición. Colex, Madrid.

HALLIDAY, S., BURNS, N., HUTTON, N., MCNEILL, F. y TATA, C. (2009) Street-level bureaucracy, interprofessional relations, and coping mechanisms: A study of criminal justice social workers in the sentencing process, Law and Policy, vol. 31, no. 4, pp. 405-428.

HANNAH-MOFFAT, K. (2005) Criminogenic needs and the transformative risk subject: Hybridizations of risk/need in penality. Punishment and Society, vol. 7, no. 1, pp. 29-53.

HANNAH-MOFFAT, K. y MAURUTTO, P. (2010) Re-contextualizing pre-sentence reports: Risk and race, Punishment and Society, vol. 12, no. 3, pp. 262-286.

LARRAURI, E. (2010) Los Programas Formativos como medida penal alternativa en los casos de violencia de género ocasional. Revista Española de Investigación Criminológica, http://www.criminologia.net/reic.html

LOWENKAMP, C., LATESSA, E. y HOLSINGER, A. (2006) The Risk principle in Action: What Have We Learned From 13,676 Offenders and 97 Correctional Programs?, Crime E Delinquency, vol. 52, n.1: 77-93.

MCIVOR, G. (2011) La participación de los jueces en la ejecución de las penas y medidas comunitarias en escocia: novedades y retos, en Larrauri, E. y Blay, E (coords) Las Penas comunitarias en Europa. Trotta, Madrid.

PERSSON, A. y SVENSSON, K. (2012) Shades of professionalism: Risk assessment in pre-sentence reports in Sweden. European Journal of Criminology, 9/2:176-191.

ROSENCRANCE, J. (1988) Maintaining the Myth of Individualized Justice: Probation Presentence Reports, Justice Quarterly, vol 5, $\mathrm{n}^{\mathrm{O}} 2: 235-256$.

RUSH, C. y ROBERTSON, J. (1987) Presentence Reports - The utility of information to the sentencing decision, Law and buman behavior, vol. 11, no. 2, pp. 147-155.

SMITH, D. (2005) Probation and Social Work. British Journal of Social Work. 35: 621-637.

TATA, C. (2010) A sense of justice: The role of pre-sentence reports in the production (and disruption) of guilt and guilty pleas, Punishment and Society, vol. 12, no. 3, pp. 239-261.

TATA, C., BURNS, N., HALLIDAY, S., HUTTON, N. y MCNEILL, F. (2008) Assisting and advising the sentencing decision process: The pursuit of 'quality' in pre-sentence reports, British Journal of Criminology, vol. 48, no. 6, pp. 835-855.

VEGAS, J. C. (2011) Modificaciones en la ejecución de la pena de trabajos en beneficio de la comunidad al amparo del Real Decreto 840/2011: un paso más hacia la administrativización de la ejecución de esta pena, Diario La Ley, nº 7743, Sección Esquemas Legales, 24 Nov. 2011, Año XXXII, Editorial La Ley.

WHITE, R. (2002) The structure and organization of criminal justice in England and Wales. An overview, McConville, M. y Wilson, G., The Handbook of the Criminal Justice Process, Oxford University Press, pp. 4-20.

\section{Sobre la autora:}

Elena Larrauri Pijoan es catedrática de Derecho penal y Criminología en la Universitat Pompeu Fabra de Barcelona. Ejerce en la actualidad de directora del grado de Criminología y Políticas Públicas de Prevención de dicha Universidad. Sus publicaciones académicas versan sobre Criminología Crítica y Violencia de Género, (Madrid, Trotta, 2007), Las Penas Comunitarias en Europa (con Ester Blay, Madrid, Trotta, 2011) y antecedentes penales (con James Jacobs: Are criminal convictions a public matter? The US and Spain, en Punishment E Society 14 (1) January 2012; y Antecedentes penales y reinserción laboral, en Revista Electrónica de Ciencia Penal y Criminología, 2011). Ha sido presidenta de la European Society of Criminology (2007-2010) y traductora de Cohen, S. (Visions of Social Control) y von Hirsh, A., (Censure and Sanctions). 